\title{
Erratum to the article Twisted Alexander polynomials and surjectivity of a group homomorphism
}

\author{
TERUAKI KITANO \\ MASAAKI SUZUKI \\ MASAAKI WADA
}

\begin{abstract}
We prove the nonexistence of surjective homomorphisms from knot groups $G\left(8_{21}\right)$, $G\left(9_{12}\right), G\left(9_{24}\right), G\left(9_{39}\right)$ onto $G\left(4_{1}\right)$ using twisted Alexander polynomials and the numbers of surjective homomorphisms onto $\operatorname{SL}(2 ; \mathbb{Z} / 7 \mathbb{Z})$.
\end{abstract}

57M25; 57M05

In [1, Section 5], we considered existence of surjective homomorphisms between knot groups. In particular, the twisted Alexander polynomial was used in order to prove nonexistence of surjective homomorphisms. To be precise, we dealt with the numerator and the denominator of twisted Alexander polynomial separately and stated that there does not exist any surjective homomorphism from $G\left(8_{21}\right), G\left(9_{12}\right), G\left(9_{24}\right)$, $G\left(9_{39}\right)$ onto $G\left(4_{1}\right)$. However, the numerator and the denominator depend on the presentation of a knot, that is, each of them is not an invariant of knots, though the twisted Alexander polynomial is. We fixed the Wirtinger presentations of knot groups and discussed the numerator and the denominator of twisted Alexander polynomial separately. By that argument, we proved only nonexistence of meridian-preserving surjective homomorphisms from $G\left(8_{21}\right), G\left(9_{12}\right), G\left(9_{24}\right), G\left(9_{39}\right)$ onto $G\left(4_{1}\right)$.

The results of [1, Section 5] are still true, which can be proved as follows. First, the number of $\operatorname{SL}(2 ; \mathbb{Z} / 13 \mathbb{Z})$-representations of $G\left(8_{21}\right)$ is 55 up to equivalence. We computed the twisted Alexander polynomials associated to them, and checked that they are not divisible by the twisted Alexander polynomial of $G\left(4_{1}\right)$ associated to the following representation $\rho$ :

$$
\begin{array}{ll}
\rho\left(x_{1}\right)=\left(\begin{array}{cc}
0 & 1 \\
12 & 8
\end{array}\right), & \rho\left(x_{2}\right)=\left(\begin{array}{ll}
1 & 1 \\
6 & 7
\end{array}\right), \\
\rho\left(x_{3}\right)=\left(\begin{array}{ll}
3 & 7 \\
2 & 5
\end{array}\right), & \rho\left(x_{4}\right)=\left(\begin{array}{cc}
10 & 2 \\
9 & 11
\end{array}\right),
\end{array}
$$


where we use the presentation of $G\left(4_{1}\right)$ on page 1322 of [1]. Therefore there does not exist any surjective homomorphism from $G\left(8_{21}\right)$ onto $G\left(4_{1}\right)$ by [1, Theorem 3.1]. We can show nonexistence of surjective homomorphisms from $G\left(9_{12}\right)$ and $G\left(9_{39}\right)$ onto $G\left(4_{1}\right)$ by similar arguments.

The number of surjective homomorphisms of $G\left(9_{24}\right)$ onto $\operatorname{SL}(2 ; \mathbb{Z} / 7 \mathbb{Z})$ is 12 up to equivalence and that of $G\left(4_{1}\right)$ is 16 . This implies the nonexistence of any surjective homomorphism from $G\left(9_{24}\right)$ onto $G\left(4_{1}\right)$, for if there were such a homomorphism, the number of surjective homomorphisms of $G\left(9_{24}\right)$ onto $\operatorname{SL}(2 ; \mathbb{Z} / 7 \mathbb{Z})$ would have to be no less than that of $G\left(4_{1}\right)$. We also tried to show nonexistence of surjective homomorphisms from $G\left(9_{24}\right)$ onto $G\left(4_{1}\right)$ using [1, Theorem 3.1], but failed within the range $p \leq 47$. For example, we fix a presentation of $G\left(9_{24}\right)$ :

$G\left(9_{24}\right)$

$=\left(\begin{array}{r|l|l}y_{1}, y_{2}, y_{3}, y_{4}, y_{5}, & y_{4} y_{2} y_{4}^{-1} y_{1}^{-1}, y_{5} y_{3} y_{5}^{-1} y_{2}^{-1}, y_{7} y_{3} y_{7}^{-1} y_{4}^{-1}, y_{2} y_{5} y_{2}^{-1} y_{4}^{-1}, \\ y_{6}, y_{7}, y_{8}, y_{9} & y_{9} y_{5} y_{9}^{-1} y_{6}^{-1}, y_{8} y_{7} y_{8}^{-1} y_{6}^{-1}, y_{3} y_{7} y_{3}^{-1} y_{8}^{-1}, y_{1} y_{8} y_{1}^{-1} y_{9}^{-1}\end{array}\right)$.

There exist $\operatorname{SL}(2 ; \mathbb{Z} / 5 \mathbb{Z})$-representations $\rho_{1}, \rho_{2}$ of $G\left(9_{24}\right), G\left(4_{1}\right)$, respectively,

$$
\begin{gathered}
\rho_{1}\left(y_{1}\right)=\left(\begin{array}{ll}
0 & 4 \\
1 & 2
\end{array}\right), \quad \rho_{1}\left(y_{2}\right)=\left(\begin{array}{ll}
4 & 1 \\
1 & 3
\end{array}\right), \quad \rho_{1}\left(y_{3}\right)=\left(\begin{array}{ll}
1 & 0 \\
0 & 1
\end{array}\right), \\
\rho_{1}\left(y_{4}\right)=\left(\begin{array}{ll}
0 & 1 \\
4 & 2
\end{array}\right), \quad \rho_{1}\left(y_{5}\right)=\left(\begin{array}{ll}
0 & 4 \\
1 & 2
\end{array}\right), \quad \rho_{1}\left(y_{6}\right)=\left(\begin{array}{ll}
0 & 1 \\
4 & 2
\end{array}\right), \\
\rho_{1}\left(y_{7}\right)=\left(\begin{array}{ll}
0 & 1 \\
4 & 2
\end{array}\right), \quad \rho_{1}\left(y_{8}\right)=\left(\begin{array}{ll}
0 & 1 \\
4 & 2
\end{array}\right), \quad \rho_{1}\left(y_{9}\right)=\left(\begin{array}{ll}
4 & 1 \\
1 & 3
\end{array}\right), \\
\rho_{2}\left(x_{1}\right)=\left(\begin{array}{ll}
4 & 4 \\
3 & 2
\end{array}\right), \quad \rho_{2}\left(x_{2}\right)=\left(\begin{array}{ll}
0 & 1 \\
4 & 1
\end{array}\right), \\
\rho_{2}\left(x_{3}\right)=\left(\begin{array}{ll}
3 & 3 \\
1 & 3
\end{array}\right), \quad \rho_{2}\left(x_{4}\right)=\left(\begin{array}{ll}
0 & 2 \\
2 & 1
\end{array}\right) .
\end{gathered}
$$

Then the numerator $\Delta_{K, \rho_{i}}^{N}$ and the denominator $\Delta_{K, \rho_{i}}^{D}$ of the twisted Alexander polynomial associated to $\rho_{i}$ are given by

$$
\begin{array}{ll}
\Delta_{9_{24}, \rho_{1}}^{N}=t^{12}+3 t^{10}+4 t^{8}+4 t^{6}+4 t^{4}+3 t^{2}+1, & \Delta_{9_{24}, \rho_{1}}^{D}=t^{2}+3 t+1, \\
\Delta_{4_{1}, \rho_{2}}^{N}=t^{4}+2 t^{3}+4 t^{2}+2 t+1, & \Delta_{4_{1}, \rho_{2}}^{D}=t^{2}+4 t+1 .
\end{array}
$$

It is easily seen that $\Delta_{4_{1}, \rho_{2}}^{N} / \Delta_{4_{1}, \rho_{2}}^{D}$ divides $\Delta_{9_{24}, \rho_{1}}^{N} / \Delta_{9_{24}, \rho_{1}}^{D}$. This does not prove nonexistence of surjective homomorphisms from $G\left(9_{24}\right)$ onto $G\left(4_{1}\right)$. Here we note that $\Delta_{41}^{N}, \rho_{2}$ does not divide $\Delta_{9_{24}, \rho_{1}}^{N}$. 
We remark the following concerning [1, Theorem 3.1]. It is not necessary to assume that $G, G^{\prime}$ are knot groups and that a surjective homomorphism has some property (eg it preserves meridian). In the proof of [1, Theorem 3.1], for the fixed the presentation of $G, G^{\prime}$, we showed that the numerators of twisted Alexander polynomial are divisible and that the denominators are same. Then we can conclude that their twisted Alexander polynomials are divisible, since twisted Alexander polynomial is independent of the choice of presentation.

\section{References}

[1] T Kitano, M Suzuki, M Wada, Twisted Alexander polynomials and surjectivity of a group homomorphism, Algebr. Geom. Topol. 5 (2005) 1315-1324 MR2171811

Department of Information Systems Science, Soka University

1-236 Tangi-cho, Hachioji-shi, Tokyo 192-8577, Japan

Department of Mathematics, Akita University

1-1 Tegata Gakuenmachi, Akita 010-8502, Japan

Department of Pure and Applied Mathematics, Graduate School of Science, Osaka University 1-1 Machikaneyama-machi, Toyonaka 560-0043, Japan

kitano@soka.ac.jp, macky@math.akita-u.ac.jp, wada@ist.osaka-u.ac.jp http://www.math.sci.osaka-u.ac.jp/ wada/

Received: 10 July $2011 \quad$ Revised: 15 September 2011 